\title{
Three-dimensional transesophageal echocardiography and cardiac magnetic resonance imaging as complementary modalities in dia- gnosis and assessment of atrial septal defect: a case presentation
}

\author{
Aleksandra Šustar* \\ Davorka Žagar \\ Ingrid Buljanović \\ Viktor Peršić \\ Thalassotherapia Opatija \\ - Clinic for treatment, \\ rehabilitation and prevention \\ of cardiovascular disease, \\ Opatija, Croatia
}

KEYWORDS: three dimensional tranesophageal echocardiography, cardiac magnetic resonance imaging, atrial septal defect.

CITATION: Cardiol Croat. 2015;10(3-4):83. | DOI: http://dx.doi.org/10.15836/ccar.2015.83

ORCID: Aleksandra Šustar, http://orcid.org/0000-0002-5983-8575 • Davorka Žagar, http://orcid.org/0000-0002-0914-6810 • Ingrid Buljanović, http://orcid.org/0000-0003-0325-2847 • Viktor Peršić, http://orcid.org/0000-0003-4473-5437

*ADDRESS FOR CORRESPONDENCE: Thalassotherapia Opatija, M. Tita 188/1, HR-51410 Opatija, Croatia. Phone:+385-91-1510-952. E-mail: aleksandramlinaric@yahoo.com

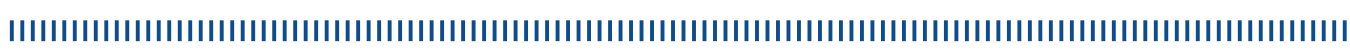

Atrial septal defect (ASD) is the most common congenital heart disease in adults and adolescents. Transthoracic echocardiography (TTE) is the primary imaging modality in diagnosing of ASD. Two dimensional transesophageal echocardiography (2D TEE) is superior to two dimensional transthoracic echocardiography (2D TTE) or three dimensional transthoracic echocardiography (3D TTE). However, 3D TEE is further superior to 2D TEE. Cardiac magnetic resonance imaging (CMR) may be useful for unusual septal defects and can provide information including shunt quantification and measurement of ventricular size and function..$^{1-3}$

This case report describes a 47-years-old man with newly diagnosed premature ventricular contractions and enlarged right heart chambers who was reffered to CMR imaging to rule out structural right ventricular disease. CMR revealed enlarged right atrium and right ventricle with mildly reduced right ventricular ejection fraction and non ishemic left ventricular hyperenhancement pattern pathognomic for myocarditis, right ventricular pressure overload (i.e. congenital heart disease) and infiltrative myocardial disease. Furthermore, cine MR imaging also revealed suspected atrial septal defect. The patient was reffered to 2D and 3D TTE and TEE. The 3D TEE confirmed sinus venosus atrial septal defect with small left to right shunt. The calculated Qp/Qs was 1.5. The patient was dissmised with antiarrhythmic and antiplatelet therapy.

Volume overloaded right ventricle might be a characteristic of both arrythmogenic right ventricular dysplasia and atrial septal defects. Considering that, patients with unexplained right ventricular volume overload should be always evaluated for possible atrial septal defect. In this case the cardiac magnetic resonance was crucial for establishing the diagnosis which was confirmed by TTE and TEE. CMR imaging is particulary useful if echocardiographic findings are technically suboptimal. However, echocardiography (TTE and TEE) is the imaging modality of choice for the diagnosis of ASD. 3D TEE enable precise measurements of ASD and may be very important for treatment.

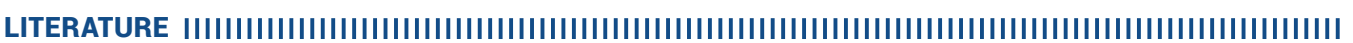

1. Roberson DA, Cui W, Patel D, Tsang W, Sugeng L, Weinert L, et al. Three-dimensional transesophageal echocardiography of atrial septal defect: a qualitative and quantitative anatomic study. J Am Soc Echocardiogr. 2011;24(6):600-10. DOI: http://dx.doi.org/10.1016/j.echo.2011.02.008

2. Kilner PJ. The role of cardiovascular magnetic resonance in adults with congenital heart disease. Prog Cardiovasc Dis. 2011;54(3):295-304. DOI: http://dx.doi.org/10.1016/j.pcad.2011.07.006

3. Sharma VK, Radhakrishnan S, Shrivastava S. Three-dimensional trans-esophageal Echocardiographic Evaluation of Atrial Septal Defects: A Pictorial Essay. Images Paediatr Cardiol. 2011;13(3):-118. PubMed: http://www.ncbi.nlm.nih.gov/pubmed/22368571
Cardiologia Croatica $\square$ 2015;10(3-4):83. 